\title{
INTELLIGENCE IN DSM-IV COMBINED TYPE ATTENTION-DEFICIT/HYPERACTIVITY DISORDER IS NOT PREDICTED BY EITHER DOPAMINE RECEPTOR/TRANSPORTER GENES OR OTHER PREVIOUSLY IDENTIFIED RISK ALLELES FOR ATTENTION- DEFICIT/HYPERACTIVITY DISORDER
}

Edmund J S Sonuga-Barke ${ }^{1 *}$, Keeley-Joanne Brookes ${ }^{4}$, Jan Buitelaar ${ }^{2}$, Paraskevi Bitsakou ${ }^{1}$, Dieter Baeyens ${ }^{3}$, Cathelijne Buschgens ${ }^{2}$, Wai Chen ${ }^{4}$, Hanna Christiansen ${ }^{5}$, Jacques Eisenberg ${ }^{6}$, Jonna Kuntsi ${ }^{4}$, Iris Manor ${ }^{7}$, Amanda Meliá ${ }^{8}$, Aisling Mulligan ${ }^{9}$, Nanda Rommelse ${ }^{10}$, Ueli Müller $^{11}$, Henrik Uebel ${ }^{12}$, Tobias Banaschewski ${ }^{13}$, Richard Ebstein ${ }^{6}$, Michael Gill ${ }^{9}$, Ana Miranda $^{8}$, Robert D Oades ${ }^{5}$, Herbert Roeyers ${ }^{3}$, Aribert Rothenberger ${ }^{12}$, Joseph Sergeant ${ }^{10}$, Hans Christoph Steinhausen ${ }^{11}$, Margaret Thompson ${ }^{1}$, Eric Taylor ${ }^{4}$, Philip Asherson ${ }^{4}$, Stephen V Faraone ${ }^{14}$

2008 American Journal of Medical Genetics, part B, 147B, 316-319

This is the reformatted manuscript submitted - prior to publication in its final form at DOI: 10.1002/ajmg.b.30596

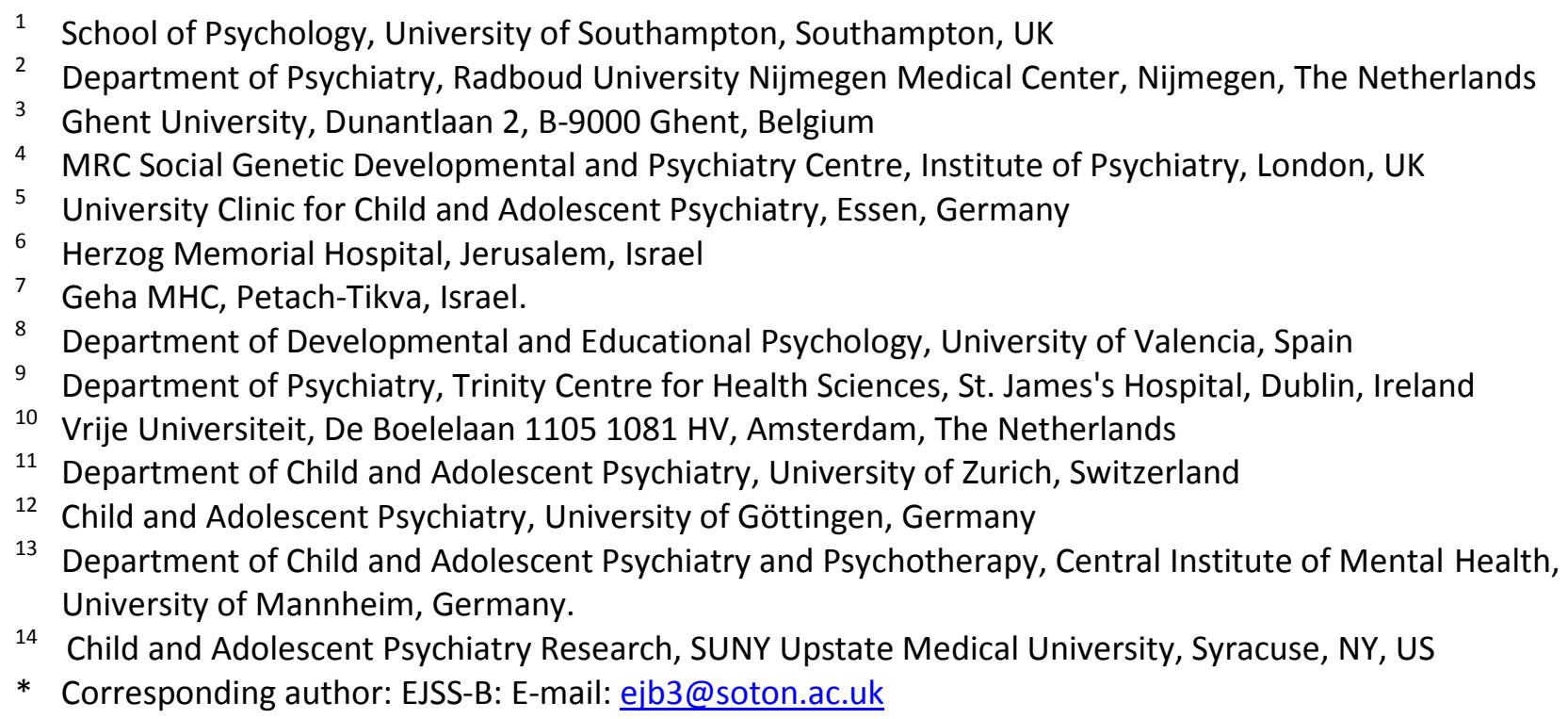

Acknowledgements: The IMAGE project is a multi-site, international effort supported by NIH grant R01MH62873 to S.V. Faraone. Site Principal Investigators are Philip Asherson, Tobias Banaschewski, Jan Buitelaar, Richard P. Ebstein, Stephen V. Faraone, Michael Gill, Ana Miranda, Robert D. Oades, Herbert Roeyers, Aribert Rothenberger, Joseph Sergeant, Edmund Sonuga-Barke, and Hans-Christoph Steinhausen. Senior co-investigators are Margaret Thompson, Pak Sham, Peter McGuffin, Robert Plomin, Ian Craig and Eric Taylor. Chief Investigators at each site are Rafaela Marco, Nanda Rommelse, Fernando Mulas, Wai Chen, Henrik Uebel, Hanna Christiansen, U. Mueller, Cathelijne Buschgens, Barbara Franke, Lamprini Psychogiou and Isabel Gabriels. Other investigators are Marieke Altink, Ellen Fliers, Ruud Minderaa and Alysa Doyle. We thank all the families who kindly participated in this research.

Funded by: NIH; Grant Number: R01MH62873

Keywords: ADHD $\bullet$ dopamine $\bullet I Q \bullet I M A G E \bullet$ genetics 


\section{ABSTRACT}

A major goal of genetic studies of Attention Deficit Hyperactivity Disorder (ADHD) is to identify individual characteristics that might help segregate the disorder's inherent heterogeneity. Mill et al. 2006 recently reported a potentially important association between two dopamine-related risk polymorphisms (DRD4 variable number tandem repeat (VNTR) in exon 3 and DAT1 VNTR in the 3' UTR) and lowered IQ in ADHD. The objective of this study was to replicate the Mill et al. ${ }^{1}$ findings in a clinical sample and to extend the analysis to a large range of alternative SNP markers of putative ADHD risk alleles identified in a recent study (Brookes et al., 2006). Participants were 1081 children and adolescents with a research-confirmed combined type ADHD diagnosis and 1300 unaffected siblings who took part in the International Multi-centre ADHD Genetics (IMAGE) project. They were recruited from multiple settings from across Europe: Belgium, Britain, Germany, Ireland, Israel, Netherlands, Spain and Switzerland. ADHD was associated with reduced IQ. However, there was no association between the two dopamine-related risk polymorphisms and IQ in either the probands or their siblings. Furthermore, other selected genetic markers previously demonstrated to be associated with ADHD in this sample were not associated with IQ. This large scale study with a clinically ascertained and rigorously diagnosed sample failed to replicate the association between genetic polymorphisms in the dopamine system and IQ in ADHD. We also observed no association of other SNPs with IQ in ADHD.

\section{INTRODUCTION:}

Attention-Deficit/Hyperactivity Disorder (ADHD) is a complex disorder with growing evidence of heterogeneity in etiology, pathophysiology and clinical expression (Sonuga-Barke 2005). One of the goals of ADHD genetic research is to investigate whether genetic factors can explain this heterogeneity, potentially identifying more homogeneous and genetically defined subgroups within the clinical phenotype in a way that could strengthen existing diagnostic approaches (Castellanos and Tannock 2002). One approach to resolving heterogeneity involves the identification of endophenotypes, intervening variables that might mediate pathways between specific genes or clusters of genes and the clinical phenotype (Stevenson et al. 2005). In this regard the replicated but small association between ADHD and functional variants in a number of genes related to dopamine neurotransmission (Faraone et al. 2005) has focused attention on the endophenotypic pathways between these genes and the clinical condition (Bellgrove et al. 2005).

Mill et al (2006) recently reported data from two epidemiologically ascertained cohorts in which children identified as meeting symptomatic criteria for ADHD and who carried high risk genotypes for the dopamine transporter (DAT1 or SLC6A3; 2 copies of the 10-repeat allele of a VNTR in the 3' UTR of the gene) and the dopamine D4 receptor (DRD4; at least one copy of the 7-repeat allele of the VNTR in exon 3), had lower IQ relative to other ADHD children in their sample. The reported effects were striking, with over a 10-point difference in IQ between the groups in the two independent datasets. This finding, suggesting as it did a genotypically defined intellectually impaired subgroup within ADHD, was potentially of considerable significance. However, the non-standard approach to diagnosis used to characterize children in one of the samples (children were identified as cases on the basis of meeting thresholds on either hyperactive/ 
impulsive or inattentive symptoms as reported by the teacher or the parent) and the very small size of the second sample (in sum 48 children with ADHD, of whom only 11 had high genotypic risk) makes replication of these results in larger samples with a full clinical work up and rigorous diagnosis essential.

Since then, Genro et al. (2006) failed to replicate the association between IQ and the Mill index of genotypic risk in three samples of clinically diagnosed Brazilian cases; 242 referred children with ADHD, 220 adults with ADHD and 100 children with ADHD inattentive type, ascertained from 12 public schools. No significant association was seen in these samples $(p=$ $0.77 ; p=0.99 ; p=0.15$ respectively). The current study attempted a further replication of the findings of Mill et al (2006) in a very large sample of children and adolescents with combined type ADHD from families recruited for the International Multicentre ADHD Genetics project (IMAGE: Asherson et al. 204). We also extended the analysis of possible genotypic markers of low IQ in ADHD by investigating the possible association with IQ of 55 Single Nucleotide Polymorphism (SNP) markers, including markers in $\mathrm{TPH} 2$, ARRB2, SYP, ADRB2, HES1, MAOA, NET and $P N M T$, identified as putative genetic risk markers for ADHD in a recent study of this sample (Brookes et al. 2006).

\section{Methods:}

\section{Sample:}

IMAGE recruited European Caucasian subjects from twelve specialist clinics in eight countries: Belgium, Germany, Holland, Ireland, Israel, Spain, Switzerland and United Kingdom. Ethical approval for the study was obtained from National Institute of Health registered ethical review boards for each centre. All ADHD probands and their siblings were aged 5 to 17 at the time of entry into the study and access was required to one or both biological parents for DNA collection. Entry criteria for probands were a clinical diagnosis of DSM-IV combined subtype ADHD and having one or more full siblings available for ascertainment of clinical information and DNA collection. Exclusion criteria applying to both probands and siblings included autism, epilepsy, $\mathrm{IQ}<70$, brain disorders and any genetic or medical disorder associated with externalizing behaviors that might mimic ADHD. The overall dataset consisted of 1246 probands and 1600 siblings. Prior to entry into the study, all probands underwent clinical evaluations by a trained clinician. Diagnostic status was confirmed using a standardized clinical assessment (see below). Wherever possible families withdrew stimulant medication for one week prior to research assessments and ratings were based on medication free periods.

For the purposes of the current analysis probands were included only if they met research criteria for combined type diagnosis (948 boys and 133 girls; mean age $=10.8$ years, SD $=2.76)$. Unaffected siblings ( 628 boys and 672 girls; mean age $=11.0$ years, $\mathrm{SD}=3.42$ ) were included in the analysis as a non-clinical, although correlated, comparison group (i.e., no diagnosis of ADHD combined, inattentive or hyperactive/impulsive). The necessary genotypic (DAT1 $\mathrm{N}=2102$ : $88 \%$ of total sample; DRD4 $\mathrm{N}=2295 ; 88.6 \%$ ) and IQ data $(\mathrm{N}=1655)$ was available for 702 ADHD cases (628 boys; mean age $=10.80$ years; $\mathrm{SD}=2.76$ ) and 694 siblings (317 boys; mean age $=10.96 ;$ SD $=3.43$ ) (table 1$)$.

\section{Attention Deficit Hyperactivity Disorder Diagnosis:}

A standardized algorithm was applied to data from the Parental Account of Childhood Symptoms (Taylor et al. 1991) to derive each of the 18 DSM-IV ADHD items, providing operational definitions 
for each behavioral symptom. The PACS is a semi-structured, standardized, investigator-based interview. A trained interviewer rates on a four point scale the frequency and severity of symptoms on the basis of parents' descriptions of the child's typical behavior in a range of specified situations (e.g. watching television, reading a book or comic, playing alone, playing with friends, going to bed, traveling). Inter-rater reliability is high with product-moment correlations for pairs of interviewers ranging from 0.79 to 0.96 . The PACS data were combined with items that scored 2 or 3 from the teacher rated Conners' (2000) ADHD subscale, to generate the total number of items from the DSM-IV symptom checklist.

\section{Intelligence testing:}

IQ was measured using Wechsler Intelligence Scales for Children (WISC-IIIR: Wechsler, 1991) which were administered to each child by centrally trained research workers using a common protocol. The vocabulary, similarities, picture completion and block design subtests from the WISC were used to obtain an estimate of the child's IQ (prorated following procedures described by Sattler (1992). Up to date, age-appropriate national population norms were available for each participating site and these were used to derive standardized estimates of intelligence on the basis of common algorithms (table 2).

\section{DNA extraction and genotyping:}

DNA was extracted directly from blood samples or cell lines at Rutgers Cell line and DNA repository in the US. In a few cases we used a mouth swab sampling technique and extracted the DNA at the SGDP laboratories in London. For genotyping of the variable number tandem repeat (VNTR) markers we used standard PCR protocols according to previous optimized protocols for the markers used in this study. SNP genotyping was undertaken by an Illumina custom SNP array in a previously reported study (Brookes et al. 2006). In this study 51 candidate genes, incorporating such systems as the dopamine and serotonin neurotransmitter systems, where screened for association with ADHD using 1038 SNP markers. Of these 1038, 55 SNP markers, in 18 genes, including $\mathrm{TPH} 2$, ARRB2, SYP, ADRB2, HES1, MAOA, NET and PNMT, had an association with ADHD with significance values of less than 0.05 . The 55 significant SNPs were investigated with QPDTPHASE (Dudbridge 2003) for association with IQ in ADHD probands only. QPDT analysis using the UNPHASED software (www.rfcgr.mrc.ac.uk/ fdudbrid/ software/unphased) estimates the covariance within each family assuming a null hypothesis of no association, with trait and genotypes being uncorrelated.

\section{Table 1}

Genotypic risk in the ADHD and unaffected siblings in terms of the presence or absence of (i) at least one DRD4 7 repeat and (ii) 10/10 DAT1

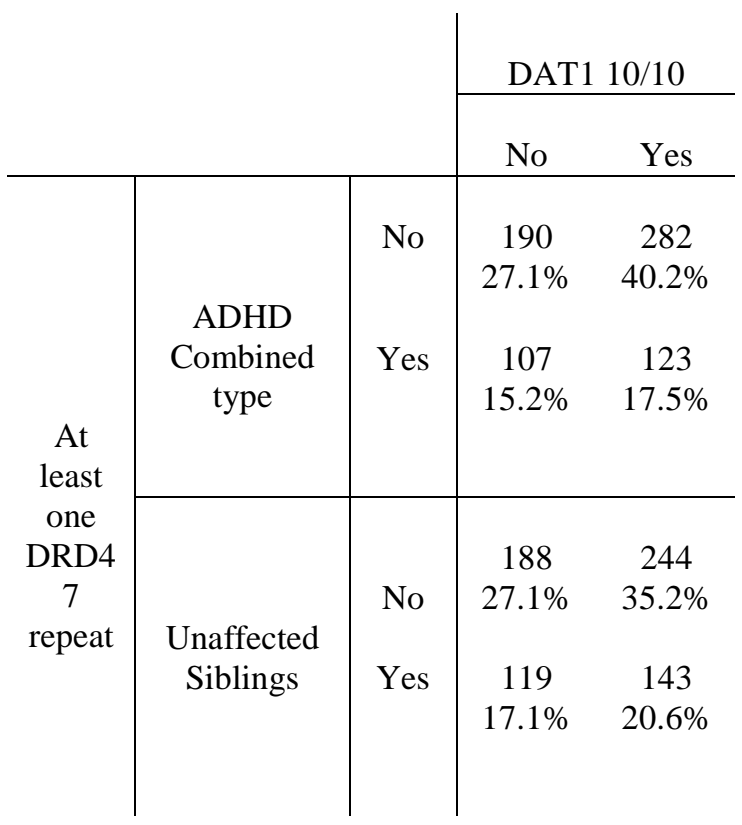

\section{RESULTS AND DISCUSSION}

As in Mill et al. (2006) ADHD was associated with lower IQ scores (ADHD mean $=100.70, S D=103.41$; Sibling mean 
$=103.41, \mathrm{SD}=103.41 ; \mathrm{t}(1653)=3.61 ; \underline{\mathrm{p}}<$ $.0001)$. This effect was especially marked for girls (female ADHD mean $=94.88, \mathrm{SD}=$ 16.16; female sibling mean $=102.74, \mathrm{SD}=$ 13.51; female $\mathrm{t}(542)=5.097 ; \mathrm{p}<.0001$; male $\mathrm{ADHD}$ mean $=101.51, \mathrm{SD}=15.97$; male sibling mean $=104.11, \mathrm{SD}=15.33$; boys $\mathrm{t}(1106)=2.60 ; \mathrm{p}<0.01)$. Table 1 reports data on the presence of DRD4 (at least one 7 repeat) and DAT1 (10/10 homozygous) risk genotypes. There was no association between the presence of DRD4 7 repeat allele and DAT1 10/10. The presence of genotypic risk in either DRD4 (ADHD probands, $F(1,710)=.14 \mathrm{p}>.7$; unaffected siblings $F(1,696)=1.32 ; p>.2)$ or DAT1 (ADHD probands, $F(1,710)=.33 p$ $>.5$; unaffected siblings $F(1,696)=1.20 ; p$ $>$.2) alone was not associated with IQ. Furthermore there was no evidence that these risks acted together either additively ( $p>.7)$ or multiplicatively $(p>$. 9) to reduce IQ. The combined genotypic risk index was not associated with IQ in either the probands or their siblings $(p>.5)$. These effects were unaffected by taking account of age, sex or comorbid oppositional defiant disorder $(p>.3)$. The Mill et al. (2006) finding was also not replicated at any of the individual sites with the current study [data available from authors on request]. When analysis was extended to children with inattentive $(\mathrm{N}=24)$ and hyperactive/impulsive subtypes $(\mathrm{N}=17)$ and to all children with ADHD combined type within the total sample (i.e. including affected siblings) not included in the main analysis but for whom IQ data was available the findings did not change. XXXXX

\section{Table 2}

IQ in the ADHD and unaffected siblings as a function of level of the dopamine genotypic risk index

Mill Index of Dopamine Genotypic Risk (standard deviations in parentheses)

\begin{tabular}{|c|c|c|c|c|}
\hline & 0 & 1 & 2 & \\
\hline ADHD & $101.8(16.3)$ & $101.6(15.0)$ & $102.9(16.3)$ & $F=0.66$ \\
\hline Combined Type & $\mathrm{N}=190$ & $\mathrm{~N}=389$ & $\mathrm{~N}=123$ & $\mathrm{P}<.51$ \\
\hline Unaffected & $103.5(13.69)$ & $103.8(14.8)$ & $13.4(143)$ & $\mathrm{F}=0.11$ \\
\hline Siblings & $\mathrm{N}=188$ & $\mathrm{~N}=363$ & $\mathrm{~N}=143$ & $\mathrm{P}<.89$ \\
\hline
\end{tabular}

We extended this analysis of genotypic markers of low IQ in ADHD (probands only) to include all 55 SNP markers shown to have nominal level of association $(P<$ 0.05) within this sample using IMAGE Illumina SNP array association study (Brookes et al. 2006). Essentially no significant correlations between IQ and genotype were observed in these extended analyses of the probands when significance levels were corrected for multiple testing. Only two markers displayed $p$-values of less or equal to 0.05 : these were both in the SLC9A9 gene (rs6793522 \& rs1900633). However these values fall well short of significance when corrected for the number of markers tested (revised alpha: $p<.009$ ). This suggested that there was no true association between IQ and the genotype of any of the SNP markers previously found to be associated with ADHD in this sample.

The field of molecular genetics is dogged by the problem of subsequent non-replication of new, interesting and 
apparently important findings. The current results together with those of Genro et al. provide strong evidence against an association between DRD4 and DAT1 risk variants and IQ in ADHD. Furthermore, in this study there was no evidence that the broader spectrum of ADHD-related genotypes mediated the association between ADHD and IQ in this sample. Given the combined statistical power of the two non-replications and their breath of ascertainment, the finding by Mill et al. may be a chance false positive. However it is important to consider differences between the studies and identify alternative explanations for differences in results between the studies. The most obvious one of these relates to the nature of the samples. In sample 1 of Mill et al., (the largest of their two samples from the British E-risk study) the cases were epidemiologically ascertained and inclusion in the ADHD group was primarily based on parent and teacher reports of symptoms of inattention and overactivity on the basis of an and/or rule (teacher or/and parent reports met DSM-IV symptom thresholds). No account was taken of impairment, a core element of normal diagnosis. This means that a broadly defined phenotype was employed with only a sub-group who would receive the clinical diagnosis of ADHD as made in the current study. One possibility is that in identifying a genetically at risk intellectually impaired subgroup in their broadly defined phenotype, they actually isolated those children who would have met the full diagnostic criteria used in the current study. This is a reasonable hypothesis since we already know that ADHD is associated with lower IQ in both clinical and general populations (Kuntsi et al. 2004).

The studies also differed in relation to exclusion on the basis of low IQ. In Mill et al. no exclusion was made and IQs ranged from 52 to 147 . In the current study children with IQs less than 70 were excluded from the study. This is common practice in studies of ADHD on the grounds that the conjunction of ADHD and low IQ may represent a qualitatively distinct presentation with intellectual impairment playing a more central, and ADHD a more peripheral role. On the basis of their findings it seems likely that those children identified as having ADHD with severe intellectual impairment were overrepresented in the high genotype risk group and perhaps contributed disproportionately to their findings. This raises the possibility that the apparent association with $\mathrm{IQ}$ in the symptomatic ADHD group may have had more to do with the genetics of intellectual impairment of which symptomatic ADHD was merely a marker.

\section{REFERENCES}

Asherson $\mathrm{P}$ and the IMAGE consortium. 2004. Attention-deficit hyperactivity disorder in the post-genomic era. European Child \& Adolesc Psychiatry; 13 (supp| 1): I50-I70.

Bellgrove MA, Hawi Z, Kirley A, Gill M, Robertson IH. 2005. Dissecting the attention deficit hyperactivity disorder (ADHD) phenotype: Sustained attention, response variability and spatial attentional asymmetries in relation to dopamine transporter (DAT1) genotype. Neuropsychologia; 43, 1847-1857.

Brookes K, Xu X, Chen W. et al. 2006. The analysis of 51 genes in DSM-IV combined type ADHD: Association signals in DRD4, DAT1 and 16 other genes. Molecular Genetics; 11: 934953.

Castellanos FX and Tannock R. 2002. Neuroscience of attention-deficit/ hyperactivity disorder: the search for endophenotypes. Nat Rev Neurosci; 3: 617-628. 
Conners CK. 2000. Conners' Rating ScalesRevised Technical Manual. North Tonawanda, New York: Multi Health Systems.

Dudbridge F. 2003. Pedigree disequilibrium tests for multilocus haplotypes. Genet Epidemiol 25:115-121.

Faraone SV, Perlis RH, Doyle AE, Smoller JW, Goralnick JJ, Holmgren MA, Sklar P. 2005. Molecular genetics of attentiondeficit/hyperactivity disorder. Biol Psychiatry; 57: 1313-1323.

Genro, JP, Roman T, Zeni CP, Grevet EH, Schmitz B, Belmonte de Abreu P, Bau CHD, Rohde LA Hutz MH (2006). No association between dopaminergic polymorphisms and intelligence variability in Attention-Deficit/ Hyperactivity Disorder, Molecular Psychiatry 11: 1066-1067.

Kuntsi J, Eley TC, Taylor A, Hughes C, Asherson P, Caspi A, Moffitt TE (2004). Co-occurrence of ADHD and low IQ has genetic origins. American Journal of Medical Genetics Part B 124B: 41-47.

Mill J, Caspi A, Williams BS, Craig I, Taylor A, Polo-Tomas $M$ et al. (2006) Prediction of heterogeneity in intelligence and adult prognosis by genetic polymorphisms in the dopamine system among children with attention-deficit/hyperactivity disorder: evidence from 2 birth cohorts. Arch Gen Psychiatry; 63: 462-469.

Sattler J M. 1992. Assessment of Children: WISC III and WPPSI-R Supplement

Sonuga-Barke EJS. 2005. Causal models of attention deficit/hyperactivity disorder: From common simple deficits to multiple developmental pathways. Biol Psychiatry; 57: 1231-1238.

Stevenson J, Asherson P, Hay D, Levy F, Swanson J, Thapar A, Willcutt E. 2005. Characterizing the ADHD phenotype for genetic studies. J Child Psych Psychiatry 8: 115-121.

Taylor E, Sandberg S, Thorley G, Giles S. 1991. The epidemiology of childhood hyperactivity. Maudsley Monograph, 33, New York: Oxford University Press.

Wechsler D. Examiner's Manual:

Wechsler Intelligence Scale for ChildrenThird Edition. Psychological Corporation: New York, 1991. 